\title{
Mental health and coping strategies in families of children and young adults with muscular dystrophies
}

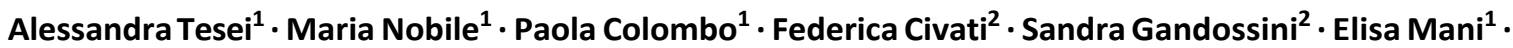 \\ Massimo Molteni ${ }^{1} \cdot$ Nereo Bresolin $^{3} \cdot$ Grazia D'Angelo $^{2}$
}

\begin{abstract}
Background Living with a progressive disease as muscular dystrophy (MD) can be challenging for the patient and the entire family from both emotional and practical point of view. We aimed to extend our previously published data about mental health in patients with MDs, also investigating coping profiles of both themselves and their parents. Furthermore, we wanted to verify whether psychological adaptation of patients can be predicted by coping strategies, taking also into account physical impairment, cognitive level and socioeconomic status.

Methods 112 patients with MDs, aged 2-32 were included. Their emotional and behavioural features were assessed through parent- and self-report Achenbach System for Empirically Based Assessment questionnaires and Strength and Difficulties Questionnaires. Development and Well-Being Assessment or Autism Diagnostic Observation Schedule were administered to confirm suspected diagnoses. Coping profile of both parents and patients was assessed through the self-administered New Italian Version of the Coping Orientation to the Problems Experienced questionnaire and its relationship with emotional/ behavioural outcome was examined in linear regression analyses.

Results High prevalence of intellectual disability and autism spectrum disorders was confirmed in Duchenne MD. Despite the high rate of internalizing symptomatology, we did not report higher rate of psychopathological disorders compared to general population. Parents tend to rely more on positive reinterpretation and less on disengagement coping. Avoidance coping, whether used by parents or patients, and ID, predicted increased emotional/behavioural problems.

Conclusions Psychosocial interventions should address problems of anxiety and depression that people with MDs frequently experience, even through fostering parents’ and childrens’ engagement coping over disengagement coping.
\end{abstract}

Keywords Muscular dystrophy · Emotional problems • Behavioural problems · Psychopathology · Coping strategies • Mental health

Maria Nobile

maria.nobile@lanostrafamiglia.it

1 Child Psychopathology Unit, Scientific Institute, IRCCS Eugenio Medea, Via Don Luigi Monza 20, Bosisio Parini, Lecco, Italy

2 Neuromuscular Unit, Scientific Institute, IRCCS Eugenio Medea, Bosisio Parini, Lecco, Italy

3 Neurology Unit, Foundation IRCCS Ca’ Granda Ospedale Maggiore Policlinico, Milan, Italy

\section{Introduction}

Muscular dystrophies (MDs) are a heterogeneous group of genetic disorders that share characteristics such as progressive muscle weakness, wasting and lack of therapy [1-3]. Although curative therapy is not yet available, in the last decades, the improvement of standard care has led to a considerable increase in patients' life expectancy [4, 5].

The most severe form of MD, Duchenne muscular dystrophy (DMD), is caused by X-linked dystrophin gene mutations and aff about 1 in 3000 males. Typically, symptoms of DMD manifest between 2 and 5 years of life, ambulation is lost by 12 years, and death mostly occurs in the second or third decade of life due to cardiac or respiratory insuffi [6]. Becker MD (BMD) is the allelic 
milder variant of the disease and aff about 1 in 20,000 males. In BMD, muscle symptoms usually have onset in the second decade, walking autonomy may be preserved up to the fi th or sixth decade, and life expectancy is not signifi y reduced, unless cardiomiopathy occurs [7].

Limb-Girdle MDs (LGMDs) include at least 8 diff ent forms of autosomal dominant (LGMD1) and more than 20 distinct forms of autosomal recessive (LGMD2) muscular dystrophies. The overall frequency varies within diff ent populations and is estimated around 20-40/10,000,000 [8, 9]. The most frequent presentation is proximal weakness that progressively impairs daily activities; respiratory and cardiac involvements are uncommon [3].

While severity, age of onset, rate of progression and consequent complications and prognosis are variable among these types of MDs [10], common features are genetic etiology, muscular involvement, disease progression, lack of risolutive therapy and physical limitations that compromise autonomy in daily life activities [11]. Along with motor impairment, neurodevelopmental and emotional/behavioural concerns are frequently associated with MDs [12-14]. This has been documented by numerous studies, which reported higher rate of internalizing symptoms, autism spectrum disorder (ASD), attention-deficit hyperactivity disorder (ADHD), intellectual disability (ID) and diffi with social functioning in children and young adults with MDs compared to general population [14-21]. As aff by chronic, progressive conditions, people with MDs need constant assistance in terms of medical, physical and emotional care and they gradually experience increased dependency on others, most often on relatives [11,22]. The increasing demands of care on multiple fronts and the physical, social and psychological hardships associated with the disease represent a potential stressor that patients and their families need to cope with and adapt to [23-25].

Caregivers of patients with MDs have been reported to experience moderate-to-high levels of stress, distress and frequent feelings of guilt, low self-esteem, sadness and depression related to the patient's condition [22, 26-29]. However, relatives with adequate coping skills, high selfesteem, and a sense of support from social network perceive lower burden and identify more positive aspects of the caregiving experience [22, 27, 30]. An interesting study conducted among relatives of MD patients revealed that most perceive their caregiving experience as having a positive impact on their lives (in terms of personal growth, resilience, altruism, sharing of the experience), all the more recognized among those who reported more practical difficulties [30]. This suggests that caregiver's perception of their experience as positive strengthens involvement and confi in their resources, preventing exceeding stress threshold, and ultimately helping to cope with their child illness and producing beneficial effects on patients in terms of quality of care received and quality of life.

Moreover, previously published studies report a good quality of life among patients with DMD, especially in the psychological health domain, despite the physical burden [31-33], supporting the hypothesis that internal resourcesof both patients and their relatives - could moderate the relationship between illness and psychological adjustment.

In its transactional model of stress and coping, within an ecological system's theory perspective, Thompson views chronic illness as a potential stressor to which the child and the family must adapt, and the relationship between illness and the psychological adjustment depends on biomedical, developmental and even psychosocial processes [34]. In this perspective, coping strategies, of parent and of individual, are hypothesized to be part of those processes moderating the illness-adjustment relationship, over and above the contributions of the illness and demographic parameters [35].

Lazarus and Folkman [36] described coping as thoughts and behaviours that people use to manage the internal and external demands of situations that are appraised as stressful.

Coping strategies have been classified conceptually into "primary control engagement coping”, "secondary control engagement coping” and "disengagement coping”. Primary control engagement coping is aimed at directly changing the stressor or the emotional reactions to the stressor. An example of primary control coping targeting the stressor is problem solving, that involves efforts to define the problem, generate alternative solutions, select the best solution and implement the solution. Primary control coping directed to emotional reactions consists in strategies such as emotional expression and emotional communication to others that aim to get moral support, sympathy, understanding and emotional relief. Secondary control engagement coping comprises eff ts to adapt to a stressor rather than change it, by strategies as acceptance (e.g., deciding to learn to leave with it) and positive reinterpretation (e.g., looking for something good in what is happening, trying to grow as a person because of the experience). Disengagement coping, conversely, is aimed at escaping from the stressor or related emotions and includes passive and maladaptive responses such as avoidance (e.g., turning to other substitute activities to think about it less), denying the stressor existence and substance use [37].

Research has shown that the use of engagement coping is related to positive health outcome and increased quality of life [37], whereas avoidant-oriented coping has been proven to be ineffective for managing long-term stress and is associated with higher psychological distress, including anxiety and depression [37-41].

Individuals differ in their propensity to rely on some coping strategies over others in response to a stressful situation [38]. However, the use of a specific coping strategy is 

a function of specific situational factors, other than dispositional, as both of them contribute to determine the subjective cognitive appraisal of threats and resources to cope with the stressor, from which derives coping behaviour. One aspect particularly relevant involved in the process of appraisal of the situation is the perceived controllability of the stressful event. Perceived control refers to the extent to which a person believes that the outcome of an event can be attributed to internal (personal) sources, external (situational/environmental) sources, or to the cause or predictability of an event [42].

Studies have shown that specific types of coping strategies are more or less effective depending upon their fitting with the appraised controllability of the situation. Primary coping is more adaptive in situations perceived highly controllable, whereas secondary coping is benefi in situations as chronic illnesses, which, even though perceived as relatively unchangeable and uncontrollable, can be appraised as a call to adapt or as a way to rediscover themselves and the others, leading to a reduction of perceived stress. The flexibility of appraisals of controllability and the flexibility of coping consequent to the appraisal could help the individual's well being and adaptation to the stressful situation, even when facing a severe chronic disease such as MD.

To our knowledge, there is only one past study conducted among patients with MDs examining the relationship between the use of certain coping strategies by parents or patients and behavioural outcomes of these latter. Thompson and colleagues [43], involving children with DMD and their relatives, found that a high use of disengagement over engagement coping by parents was associated with higher levels of both internalizing and externalizing behavioural problems in children. Consistently, more recent studies conduced on other chronic conditions (e.g., diabetes, chronic pain, cancer) have shown substantial evidence that disengagement coping is related to poorer adjustment [40].

In the present study, we aim to (1) extend our previous published data on prevalence of emotional, behavioural and neurodevelopmental problems of children with DMD [15], through the inclusion of other forms of MDs, namely BMD and LGMD together with DMD, and through the extension of age range (we recruited children and young adults until 32 years of age). Because of the progressive nature of the diseases, extending the age range can allow to observe the characteristics of emotional-behavioural profi in relation to increased levels of physical impairment and clinical severity and complexity. The inclusion of different forms of MDs, on the other hand, allows to observe the prevalence of emotional and neurodevelopmental disorders across MDs that have different levels of central nervous system (CNS) involvement (more severe for DMD, milder for BMD, typically absent for LGMD); (2) verify whether there are significant differences in the coping styles of parents and patients when compared to published general population norms. To our knowledge, the only Italian tool on coping having published norms able to be compared was validated in an adult sample; this constrained our investigation of self-report coping styles, because, to make balanced comparisons, we needed to exclude children. We, therefore, involved all subjects older than 16 , as at this age, logical reasoning, planning and other high-order thinking are considered at adult level of maturity [44]; (3) examine the role of parental coping strategies in relation to emotional/behavioural problems of their children; (4) examine the role of patients' coping strategies in relation to their self-reported emotional/behavioural problems. In achieving the latter two aims, the contribution of patients' physical condition and cognitive level will be also taken into account.

It was hypothesized that patients with MDs would display — compared to general population - higher prevalence of neurodevelopmental disorders (i.e., ID, ASD, ADHD), especially for those MDs with a higher involvement of CNS (DMD and BMD). Based on our previous study [15], it was also hypothesized the presence of a higher rate of reported emotional/behavioural symptoms that, however, would not reach diagnosis level. Looking at coping styles, it was hypothesized that both patients and parents would havecompared to general population-a coping profile that would involve a greater use of acceptance and a lower use of problem solving, as typically happen when people adapt to relatively uncontrollable stressors. Finally, we hypothesized that increased physical/cognitive impairment and the use of avoidance strategies would be associated with increased emotional/behavioural problems in patients.

\section{Materials and methods}

\section{Participants}

A clinical population of 609 patients with inherited neuromuscular disorders, comprising, among these, 133 with DMD (age range 2.8-32), 56 with BMD (age range 7-63) and 70 with LGMD (age range 9-73) attends the Neuromuscular Unit of Scientifi Institute IRCCS E.Medea for periodic clinical assessment. Among them, a total of 115 responding to the inclusion criteria (described below) were invited to take part to the study during their attendance at the Institute as in patients or outpatients. A total of 113 including families or individual patients in case of adults living without parents agreed to participate in the research. The fi sample consisted of 112 patients who returned questionnaires. Inclusion criteria were (1) confirmed clinical and molecular diagnosis of DMD, BMD and LGMD, according to international criteria [45-47], (2) age $\leq 32$ years old, (3) 
comprehension of the procedures and aims of the study, (4) signature of a written informed consent form (obtained from parents for patients younger than 18 years).

The study was approved by the Ethical Committee of the 'E. Medea' Scientific Institute according to the Declaration of Helsinki.

\section{Procedure}

To assess cognitive, emotional and behavioural features, intelligent test was administered to patients and-if aged at least 11-self-report screening questionnaires about their emotional/behavioural problems were proposed. Parents completed the parallel version of the questionnaires, answering questions about their child emotional/behavioural problems. Patients exceeding clinical cut-offs on screening questionnaires were subsequently evaluated with a clinical structured interview, using its parent, adolescent or adult version. Preschool patients with elevated autism spectrum problems based on questionnaires and patients with a clinical diagnosis of ASD based on diagnostic and statistical manual of mental disorders (DSM) criteria were further assessed with structured observation based on the Autism Diagnostic Observation Schedule (ADOS).

Coping strategies were examined with specific self-report questionnaires completed by both parents and children (if older than 16 years of age), regarding their own dispositional coping styles.

Name of each questionnaire and diagnostic tool, contents and tested sample are illustrated in Fig. 1.

\section{Measures administered to patients}

\section{Demographicand clinical characteristics}

Demographic features including participants' age, family structure, and family socioeconomic status (SES) measured using Hollingshead nine-point occupation scale [48] were collected. Clinically relevant information such as walking ability (classifying patients as ambulant or wheelchair
Fig. 1 Name, content and tested sample of questionnaires and diagnostic tools. YSR 11-18 Youth Self-Report (11-18 years), CBCL 1.5-5 Child Behaviour Checklist (1.55 years), CBCL 6-18 Child Behaviour Checklist 618 years, ASR 18-59 Adult Self-Report $18-59$ years, ABCL 18-59 Adult Behaviour Checklist $18-59$ years, $S D Q$ 11-18+ Strength and Difficulties Questionnaire 11-18 years or more, SDQ 2-18+ Strength and Difficulties Questionnaire 2-18 years or more, $D A W B A$ Development and Well-Being Assessment, COPE-NVI Coping Orientation to the Problems Experienced-New Italian version, ADOS Autism Diagnostic Observation Schedule

\begin{tabular}{|c|c|c|c|}
\hline \multicolumn{4}{|c|}{ Informant-based tools } \\
\hline \multicolumn{4}{|c|}{ Patients psychological status } \\
\hline & Informant & Tested sample & Contents \\
\hline YSR 11-18 & Self & 31 children with MDs & \multirow{3}{*}{$\begin{array}{l}\text { Internalizing (anxiety, depression, somatic } \\
\text { complaints) and externalizing } \\
\text { (aggressive/rule breaking behaviour) } \\
\text { symptoms, attention problems, thought } \\
\text { problems, social problems }\end{array}$} \\
\hline CBCL 1.5-5 & Parent & 6 children with MDs & \\
\hline CBCL 6-18 & Parent & 55 children with MDs & \\
\hline ASR 18-59 & Self & $\begin{array}{l}50 \text { young adults with } \\
\text { MDs }\end{array}$ & \multirow{2}{*}{$\begin{array}{l}\text { Internalizing (anxiety, depression, somatic } \\
\text { complaints) and externalizing (aggressive/rule } \\
\text { breaking behaviour) symptoms, attention } \\
\text { problems, thought problems, intrusive } \\
\text { behaviour }\end{array}$} \\
\hline ABCL 18-59 & Parent & $\begin{array}{l}29 \text { young adults with } \\
\text { MDs }\end{array}$ & \\
\hline SDQ 11-18+ & Self & $\begin{array}{l}78 \text { children and young } \\
\text { adults with MDs }\end{array}$ & \multirow{2}{*}{$\begin{array}{l}\text { Emotional problems, conduct problems, } \\
\text { hyperactivity-inattention, peer problems, } \\
\text { prosocial behaviours, overall distress and } \\
\text { impairment }\end{array}$} \\
\hline SDQ 2-18+ & Parent & $\begin{array}{l}57 \text { children and young } \\
\text { adults with MDs }\end{array}$ & \\
\hline DAWBA & Parent/Self & $\begin{array}{l}31 \text { children and young } \\
\text { adults with MDs }\end{array}$ & $\begin{array}{l}\text { Questions exploring psychiatric diagnoses } \\
\text { based on the DSM-V and ICD } 10 \text { criteria }\end{array}$ \\
\hline \multicolumn{4}{|c|}{ Coping strategies } \\
\hline COPE-NVI & Self & $\begin{array}{l}51 \text { children and young } \\
\text { adults with MDs; } \\
79 \text { parents }\end{array}$ & $\begin{array}{l}\text { Use of social support, avoidance strategies, } \\
\text { positive attitude, problem solving, turning to } \\
\text { religion when exposed to stress }\end{array}$ \\
\hline \multicolumn{4}{|c|}{ Observation-based tools } \\
\hline \multicolumn{4}{|c|}{ Diagnosis of Autism Spectrum Disorders (ASD) } \\
\hline ADOS & & 7 children with MDs & $\begin{array}{l}\text { Assesses communication, reciprocal social } \\
\text { interaction, imagination/creativity, } \\
\text { stereotyped behaviours and restricted } \\
\text { interests to inform the diagnosis of ASD }\end{array}$ \\
\hline
\end{tabular}


bound), ongoing therapies and the presence of heart dysfunction were collected for each group of patients.

\section{Cognitive assessment}

Cognitive ability was assessed using the Wechsler Intelligence scales or Griffi hs scales as required based on the patient's age.

\section{Psychological status: screening questionnaires}

Emotional and behavioural problems with their impact on patients' everyday life were assessed by Achenbach System for Empirically Based Assessment (ASEBA) [49, 50] questionnaires and Strength and Difficulties Questionnaire (SDQ) [51]. The ASEBA comprises a family of instruments widely adopted in research and clinical practice measuring emotional/behavioral problems, which are sorted in internalizing, externalizing and total problems broadband scales, and narrowband scales (cross-informant syndrome scales). Children from 11 to 18 years can complete themselves the Youth Self-Report (YSR) form. Adults from age 18 to 59 can be assessed with the self-report questionnaire Adult SelfReport (ASR). According to ASEBA multicultural manual, $t$ scores greater than 69 for syndrome scales and greater than 63 for broadband scales (internalizing, externalizing and total problems) were considered to be in the clinical range. Age-appropriate ASEBA self-report form was given to patients. In our sample, the majority of YSR/ASR scales showed Cronbach's $\alpha$ coefficients ranging from 0.88 to 0.61 , except four YSR scales (Somatic Complaints, Social Problems, Thought problems, Rule-Breaking behaviour) and two ASR scales (thought problems, intrusive behaviour) with an $\alpha$ coefficient $<0.60$.

SDQ is a briefer comprehensive questionnaire, which has been used as a screening tool for child psychopathology in both community and clinical settings [52]. It consists of 25 items, covering emotional problems, conduct problems, hyperactivity-inattention, peer problems and prosocial behaviours. The SDQ also include an impact supplement to assess chronicity, overall distress, social and educational impairment and burden on others of the reported problems. The impact score is made up by one item asking about perceived level of distress, and four items asking about the interference that the person's emotional/ behavioral problems have with family life, friendships, work or study and leisure activities. SDQ exists in several versions, for different informants (parent, teacher, self) and age ranges (4-17 years old, 11-17 years old, 18 years or more). For each scale, score can be categorized as 'abnormal' if it exceeds a specific clinical cut-point [53]. Similar to ASEBA questionnaires, the self-report version of SDQ was given for patients aged 11 and over. In our sample, Cronbach's $\alpha$ coeffi of self-report SDQ scales ranged from 0.78 to 0.62 , except for Conduct problems and Peer problems scales $(\alpha<0.60)$.

\section{Assessment of prevalence of psychiatric disorders based on astructured clinical interview or protocol observation}

The clinical assessment was conducted using the Development and Well-Being Assessment diagnostic interview (DAWBA) [54] or the Autism Diagnostic Observation Schedule (ADOS) [55].

The DAWBA combines a structured part and a semistructured part, and it is designed to generate present-state psychiatric diagnoses based on the DSM-V and ICD 10 criteria for children, adolescents and adults. The structured sections explore the following psychopathological areas: separation anxiety, simple phobia, social phobia, panic disorder with/without agoraphobia, post-traumatic stress disorder, obsessive-compulsive disorder, generalized anxiety disorder, major depression, ADHD, behavioural disorder, and less common disorders. The semistructured part of the interview elicits a verbatim account of any reported problems. The DAWBA has satisfactory validity and inter-rater reliability $[54,56]$. The answers to the structured questions from the interviews and questionnaires were fed into a computerized diagnostic algorithm. This algorithm provided six levels of prediction of the probability of a disorder, ranging from very unlikely to probable, the "DAWBA Bands." The subject is assigned to one of six probability bands corresponding to the approximate prevalence in an epidemiological sample [57] ranging from less than $0.1 \%$ likely to more than $70 \%$ likely (the other thresholds are $0.5 \%$, $3 \%$, $15 \%$, and $50 \%)$. Defi e diagnosis was assigned relying on both provisional diagnoses generated by computer and transcripts of answers to open-ended questions. The interviews were administered by a trained interviewer to patients aged 11 and over exceeding clinical cut-off on screening questionnaires.

The ADOS is a standardized, semistructured observational assessment used to assess communication, reciprocal social interaction, imagination/creativity, and stereotyped behaviours and restricted interests to inform the diagnosis of ASD. The ADOS is organized into four modules based on the individual's chronological age and expressive language level, ranging from preverbal to verbally fluent. All ADOS assessments were administered and scored by a licensed clinical child psychiatrist. 


\section{Coping strategies}

Coping strategies were evaluated with the New Italian Version of the Coping Orientation to the Problems Experienced (COPE-NVI), a self-report measure composed of 60 items $[58,59]$ in which respondents were asked to indicate, on a four-point scale, how often they used a specific coping strategy when they experience substantial stress. Response choices are the following: "I usually do not do this at all", "I usually do this a little bit", "I usually do this a medium amount", and "I usually do this a lot". Results of confirmatory factor analysis (CFA) indicated five basic dimensions of the instrument: (1) social support, refers to the seeking of instrumental help or emotional comfort, (2) avoidance strategies, includes avoidant coping responses such as denial and behavioural, mental or alcohol-drug disengagement, (3) positive attitude, measures acceptance and positive reinterpretation of the event, (4) problem solving, refers to the use of active, problem-focused strategies such as planning and suppression of competing activities, (5) turning to religion refers to relying on religion and lack of humour. We asked patients if older than 16 years of age to complete the questionnaire. In this subsample, Cronbach's $\alpha$ coefficients ranged from 0.88 to 0.75 across the five COPE scales.

\section{Measures administered to parents}

\section{Psychological status of children:screening questionnaires}

ASEBA questionnaires Child Behaviour Checklist (CBCL) for ages 1.5-5 (CBCL 1.5-5) and for ages 6-18 (CBCL 6-18) are designed to be completed by parents of preschool or school-age children, respectively; ASEBA Adult Behaviour Checklist for ages 18-59 (ABCL 18-59) can be completed by parents of children aged 18 and over. SDQ also has its parentreport versions, that can be completed by parents of preschool (2-4 years old), school-age (4-17 years old) or adult (aged 18 and over) children. These tools allow exploring children emotional/behavioural problems from parents' point of view and are structured as their self-report counterparts described above in terms of scales and cut-off scores.

Age-appropriate ASEBA and SDQ parent-report version was given to parents. In our sample, the majority of CBCL/ ABCL scales showed Cronbach's $\alpha$ coeffi ranging from 0.88 to 0.65 , except three CBCL scales (somatic complaints, thought problems, rule-breaking behaviour) and four ABCL scales (thought problems, rule-breaking behaviour, intrusive behaviour and total problems) with an $\alpha$ coeffi $<0.60$. Cronbach's $\alpha$ coeffi of parentreport SDQ scales ranged from 0.75 to 0.62 , except for conduct problems, peer problems and prosocial behaviour scales $(\alpha<0.60)$.

\section{Assessment of prevalence of psychiatric disorders of children based on a structured clinical interview or protocol observation}

The parent-report version of DAWBA interviews was administered by a trained interviewer to parents of patients exceeding clinical cut-offs on screening questionnaires.

\section{Coping strategies of parents}

Parental coping strategies were also evaluated with the aforementioned COPE-NVI. We asked parents to complete the questionnaire. In this subsample, Cronbach's $\alpha$ coefficients ranged from 0.87 to 0.72 across the five COPE scales.

\section{Data analysis}

Statistical analysis plan is reported in Fig. 2.

Descriptive statistics were generated for demographic and clinical variables and are reported as mean and standard deviations (SDs) values for continuous variables and frequencies/rates for categorical variables.

For the analysis of emotional/behavioural profi we classified patients in two groups: children (those up to and including 18 years) with their corresponding parent-report measures (CBCL, parent-report SDQ) and adults (those over 18 years), with their corresponding self-report measures (ASR, self-report SDQ). Means and SDs are reported for each scale. The association between SDQ and ASEBA questionnaires was assessed by the Pearson correlation coeffi Correlations between ASEBA internalizing scale score and the internalizing SDQ scales scores (emotional problems and peer problems) were moderate to strong (ranging from $r=0.36$ to 0.71 ). A similar pattern was observed for correlations between ASEBA externalizing scale and SDQ conduct problems scale, which ranged from $r=0.58$ to 0.61 . ASEBA attention problems scale showed strong correlations with SDQ hyperactivity scale (ranging from $r=0.73$ to 0.74 ).

One-sample $t$ tests were performed to compare mean COPE scores of our samples with published Italian norm means [59].

Linear regression analyses were performed to assess the association between coping strategies (COPE scores) and psychopathological symptoms from two perspectives: parental coping strategies in relation to children emotional/behavioural problems derived from parent-report measures CBCL 
Fig. 2 Statistical analysis plan

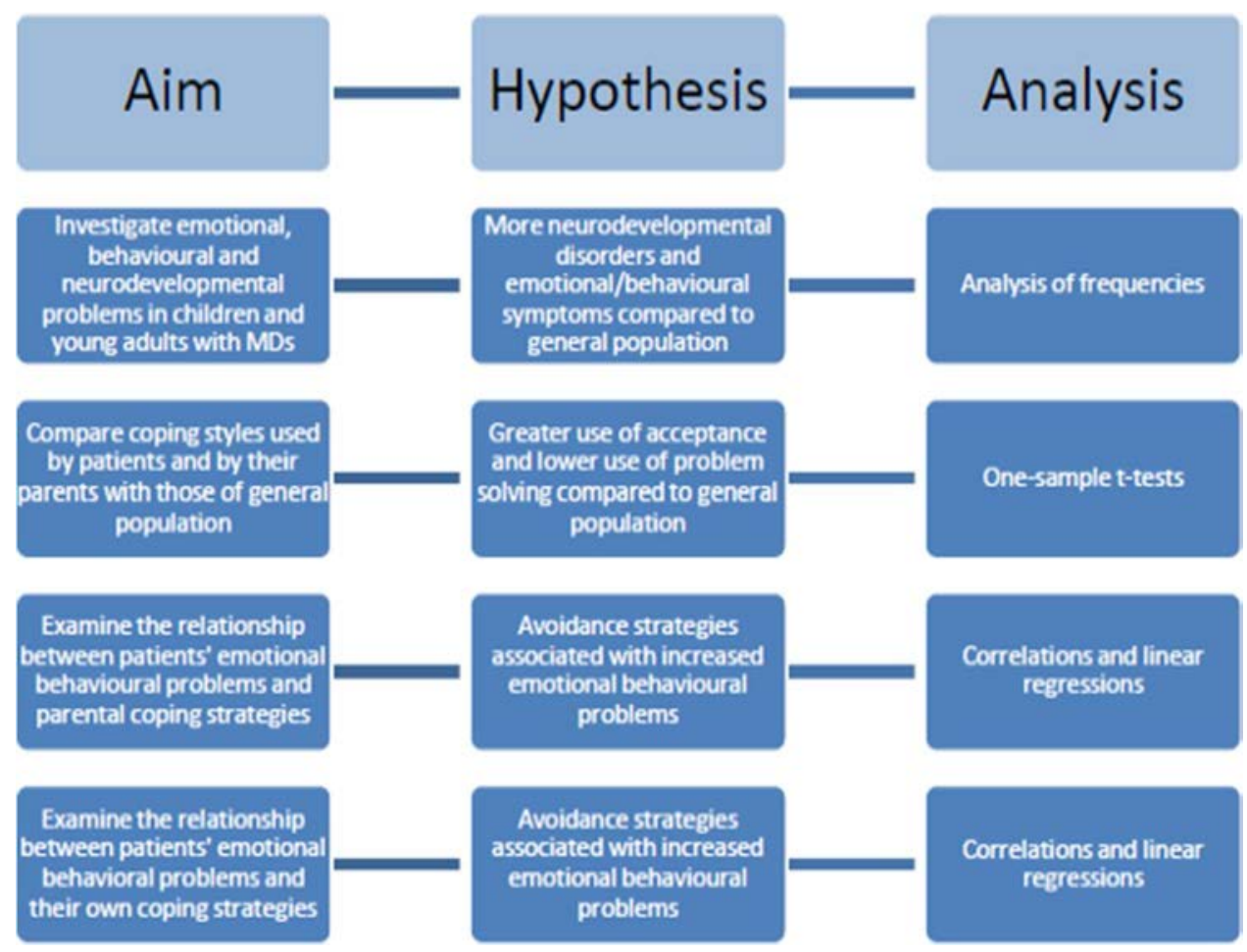

or ABCL (age range 2-31) and patients' coping strategies in relation to their own self-reported emotional/behavioural problems derived from YSR and ASR (age range 17-32). ASEBA Syndrome Scales $T$ scores were used as outcome measures, whereas COPE scales scores were entered as independent variables. In each regression, we included among independent variables only those COPE scales that showed a significant $(p<0.05)$ or a near-significant $(p \leq 0.07)$ correlation with a given syndrome scale at the Pearson correlation test (see Supplementary Table 1 and Supplementary Table 2). Similarly, physical impairments, i.e., wheelchair use and cardiac function, were entered as additional independent variables on the basis of previous correlation analysis results. Individual variables age and ID were included by default in the analyses as independent variables. We chose to include in the regression analyses ASEBA rather than SDQ on the basis of the good comparability between measures and of the larger spectrum of emotional/behavioural problems that can be identified by ASEBA.

Bonferroni's correction was used to adjust for multiple comparisons.

\section{Results}

\section{Descriptive results, analysis performed on patients' sample}

\section{Demographic/clinical characteristics and cognitive assessment}

Distribution of diagnoses, clinical, demographic features (gender, age, family status, SES) and cognitive level among the 112 patients completing the study are reported in Table 1. Most patients had DMD (75\%), followed by BMD (15.2\%) and LGMD (9.8\%). The mean age of participants was 17.24 (SD 7.46, range 2-32 years). Families of enrolled patients were mainly biparental (82.1\%) and they had a medium SES.

At the time of assessment, 72 patients (74.3\%) were wheelchair bound, 57 (50.9\%) had a cardiomyopathy and, considering the 84 DMD patients, 31 were under current steroid therapy.

The mean full-scale intelligence quotient (FSIQ) was 86.47, with an estimated $22.3 \%(n=25)$ of ID (i.e., IQ score below 70); all boys with ID were affected by DMD and they represented $27.9 \%$ of the subsample younger than 19 years and $15.7 \%$ of the subsample older than 18 years. 
Table 1 Clinical and demographic features of the sample $(N=112)$

\begin{tabular}{|c|c|}
\hline & Mean (SD) \\
\hline \multicolumn{2}{|l|}{ Age } \\
\hline Total & $17.24(7.46)$ \\
\hline DMD & $16.01(7.34)$ \\
\hline BMD & $20.24(7.61)$ \\
\hline LGMD & $22(5.04)$ \\
\hline VIQ & $87.68(16.90)$ \\
\hline PIQ & $91.21(17.64)$ \\
\hline \multirow[t]{2}{*}{ FSIQ } & 86.47 (19.68) \\
\hline & $N(\%)$ \\
\hline \multicolumn{2}{|l|}{ Age range } \\
\hline Children (2-18 years) & $61(54.50)$ \\
\hline Adults (over 18 years) & $51(45.50)$ \\
\hline \multicolumn{2}{|l|}{ Phenotype } \\
\hline DMD & $84(75.00)$ \\
\hline BMD & $17(15.20)$ \\
\hline LGMD & $11(9.80)$ \\
\hline \multicolumn{2}{|l|}{ Gender } \\
\hline Males & $108(96.40)$ \\
\hline Females & $4(3.60)$ \\
\hline Intellectual disability & $25(22.3)$ \\
\hline Wheelchair & $72(74.30)$ \\
\hline Cardiomyopathy & $57(50.90)$ \\
\hline \multirow[t]{2}{*}{ Steroid (only among 84 DMD) } & $31(36.90)$ \\
\hline & Mean (SD) \\
\hline \multirow[t]{2}{*}{ Socioeconomic status (SES) } & 48.29 (19.89) \\
\hline & $N(\%)$ \\
\hline \multicolumn{2}{|l|}{ Family structure } \\
\hline Two parents & $92(82.10)$ \\
\hline Single parent & $13(11.60)$ \\
\hline Reconstituted family & $3(2.70)$ \\
\hline Live alone & $4(3.60)$ \\
\hline
\end{tabular}

SES socio economic status, VIQ verbal intelligence quotient, PIQ performance intelligence quotient, FSIQ full-scale intelligence quotient, $D M D$ Duchenne muscular dystrophy, BMD Becker muscular dystrophy, $L G M D$ limb girdle muscular dystrophy

\section{ASEBA and SDQ}

For one adult subject (age 19+), we did not have the selfreport scores, as he returned only the parent-report form.

In Table 2, we report mean and SD of CBCL $(N=61$, age 2-18) and ASR ( $N=50$, age $19+)$ syndrome and broadband scales $t$ scores. In Table 3 are reported mean and SD of SDQ parent- ( $N=57$, age $2-18)$ and self-report $(N=50$, age $19+)$ scale scores. To explore which behavioural outcomes were "clinically relevant”, prevalence and rate of clinical cases for each scale were also reported. Among children, highest CBCL means emerged for withdrawn/depressed and social problems scales. Looking at broadband scales, a remarkable $24.59 \%$ scored in the clinical range for internalizing problems, which is a high rate compared to Italian normative data $(9.8 \%$ of CBCL caseness according to Frigerio and colleagues [60]). On the contrary, we found very low prevalence of externalizing problems at clinical range (1.64\%). Parent completed SDQ mean scores and frequencies of emotional behavioural problems were in line with published data regarding European sample of similar age range [61-63] with the exception of Peer problems scale (17.54\% classified as clinical), which showed higher rate of reported symptomatology.

In the young-adult subsample, ASRs profi resembles that of CBCLs as the highest mean score was showed by an internalizing subscale, i.e., Anxious/depressed scale. A high frequency of internalizing problems at clinical range was replicated (18\%) and no cases were classified as clinical for externalizing problems, in accordance with prevalence rate of self-reported SDQ clinical conduct problems (0\%). A slight elevation of mean score was observed for ASR Attention problems scale, where $2 \%$ of young adults fell in the clinical range; this same percentage of clinical cases was also observed for SDQ Hyperactivity scale.

\section{DAWBA and ADOS}

Assessment with the clinical structured interview DAWBA was performed on 31 patients out of the total number of 112 (27.68\%; 13.4\% children, $14.4 \%$ young adults) who exceeded clinical cut-off on screening questionnaires. The prevalence of patients actually diagnosed with an emotional and behavioural disorder according to DAWBA was $12.50 \%$ (11.48\% among children, $13.72 \%$ among young adults). In the subsample of children, externalizing disorders were slightly more frequent than internalizing disorders $(4.92 \%$ of ADHD and $1.64 \%$ of oppositional disorders versus $4.92 \%$ of anxiety disorders).

Based on protocol criteria, ADOS assessment was conducted with seven children. All of them were DMD and met criteria for ASD, representing $11.48 \%$ of the children sample (13.46\% of DMD children).

In the young-adult subsample, internalizing disorders were six times more prevalent than externalizing disorders (11.76\% of anxiety and depressive disorders versus $1.96 \%$ of ADHD); no cases of conduct/oppositional disorder or ASD were found.

Considering DMD, the overall rate of psychopathology was 9.64\%. Within the adult DMD subsample, the frequency of psychopathology was $12.5 \%$ whereas among DMD children was 7.7\%. Regarding the other type of MDs examined, the overall rate of psychopathology was $23.53 \%$ within BMD and 18.18\% within LGMD. 
Table $2 T$ scores (expressed as mean and SD) and prevalence (i.e., number of cases exceeding clinical cut-off) of emotional behavioural problems from ASEBA questionnaires syndrome and broadband (internalizing, externalizing and total problems) scales

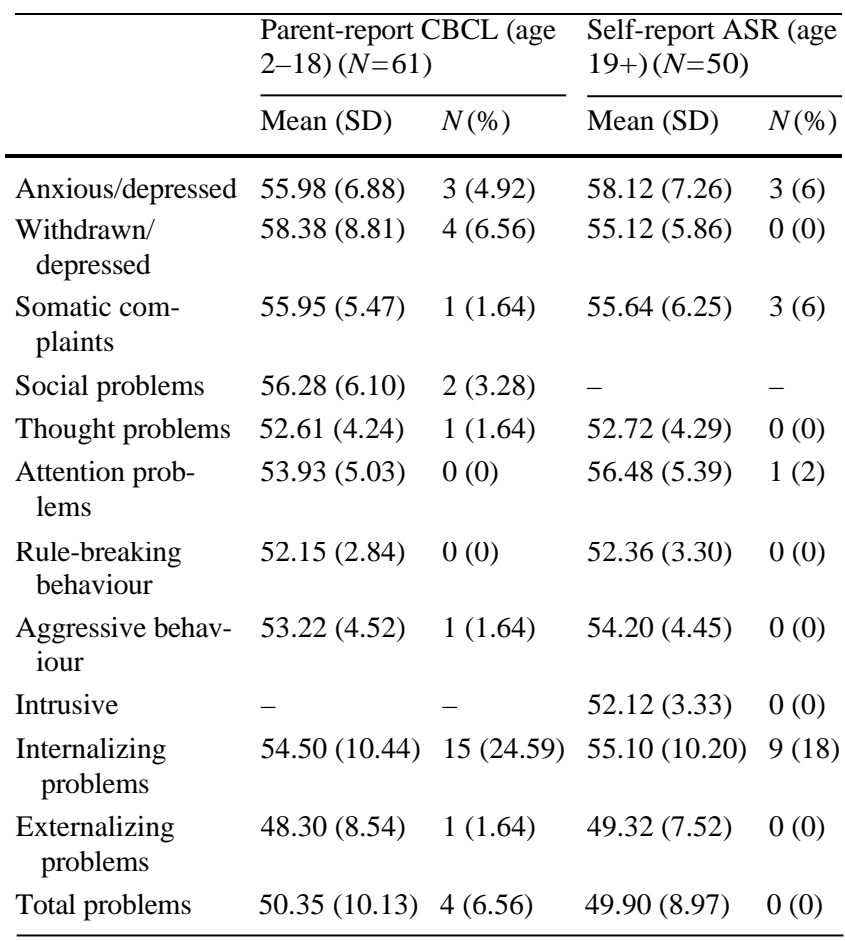

According to ASEBA multicultural manual, clinical scores correspond to $T>69$ for syndrome scales and $T>63$ for broadband scales $C B C L$ child behaviour checklist, $A S R$ adult self-report

Table 3 Raw scores and prevalence (i.e., number of cases exceeding clinical cut-off) of emotional behavioural problems from SDQ questionnaires

\begin{tabular}{lllll}
\hline & $\begin{array}{l}\text { Parent completed SDQ } \\
\text { (age 2-18) }(N=57)\end{array}$ & $\begin{array}{l}\text { Self completed } \\
\text { SDQ (age 19+) } \\
(N=50)\end{array}$ \\
\cline { 2 - 5 } & Mean (SD) & $N(\%)$ & Mean (SD) & $N(\%)$ \\
\hline Emotional problems & $1.84(1.99)$ & $6(10.53)$ & $2.66(2.44)$ & $4(8)$ \\
Conduct problems & $1.57(1.50)$ & $6(10.53)$ & $1.49(1.23)$ & $0(0)$ \\
Hyperactivity & $2.37(2.25)$ & $3(5.26)$ & $2.28(1.94)$ & $1(2)$ \\
Peer problems & $1.84(1.85)$ & $10(17.54)$ & $1.36(1.48)$ & $0(0)$ \\
Prosocial & $7.67(1.56)$ & $1(1.75)$ & $7.87(1.91)$ & $3(6)$ \\
Total difficulties & $7.43(5.22)$ & $2(3.51)$ & $7.79(5.39)$ & $1(2)$ \\
Impact & $0.35(0.85)$ & $7(12.3)$ & $0.45(1.16)$ & $6(12)$ \\
\hline
\end{tabular}

Clinical range scores are as follows: emotional problems: 5-10; conduct problems: 4-10; hyperactivity: 7-10; peer problems: 4-10; prosocial: 0-4; total difficulties: 17-40; impact: 2-10

$S D Q$ Strength and Difficulties Questionnaire

\section{Coping strategies}

Descriptive analyses for cope scales and statistical comparisons between patients $(N=51)$ and previously published Italian data from Sica and colleagues [59] on general community sample are presented in Table 4.

Patients diff ed from general community as they used less problem solving strategies and lower turning to religion; these differences were still significant after Bonferroni correction; for the other dimensions of COPE patients did not significantly differ from general population.

\section{Descriptive results, analysis performed on parents' sample}

\section{Coping strategies}

Descriptive analyses for cope scales and statistical comparisons between caregivers $(N=79)$ and previously published Italian data from Sica and colleagues [59] on general community sample are presented in Table 4.

Compared to general community, caregivers of patients with MDs relied less on social support, avoidance and problem solving while they had a higher level of positive attitude. After Bonferroni correction, we observed still signifi differences for avoidance and problem solving scales.

\section{Correlational analyses}

Supplementary Table 1 shows results of correlations between ASEBA parent-report syndrome scales (CBCL/ $\mathrm{ABCL}$ ) and age, SES, physical/cognitive impairment and parents' COPE scales $(N=76)$. Supplementary Table 2 shows results of correlations between ASEBA self-report syndrome scales (ASR/YSR) and age, SES, physical/cognitive impairment and patients' COPE scales $(N=51)$. Based on these results, regression analysis concerned only those syndrome scales showing a signifi $\quad(p<0.05)$ or a near-signifi $(p \leq 0.07)$ correlation with any COPE scale.

\section{Multivariate analyses}

\section{Regressions performed for patient coping}

Significant results from multiple linear regression analysis examining the contribution of patients' own coping strategies on their emotional behavioural problems $(N=51)$ are presented in Table 5.

The first model statistically significantly predicted anxious/depressive symptoms, explaining $28.1 \%$ of the variance. The third model significantly predicted internalizing 
Table 4 Mean and standard deviation for the five dimensions of COPE NVI in our groups of caregivers $(N=79)$ and patients $(N=51)$ compared to general community sample, composed by 457 individuals

\begin{tabular}{llll}
\hline & $\begin{array}{l}\text { General population } \\
\text { sample } N=457^{\mathrm{a}}\end{array}$ & COPE NVI patient $N=51$ & COPE NVI parent $N=79$ \\
\hline COPE NVI & & & \\
Social support & $27.7(8.4)$ & $26.80(6.86)$ & $26.03(7.22)^{*}$ \\
Avoidance strategies & $23.5(5.1)$ & $23.57(5.05)$ & $21.73(4.90)^{* *}$ \\
Positive attitude & $30.9(6)$ & $31.43(6.27)$ & $32.38(5.96)^{*}$ \\
Problem solving & $32(6.7)$ & $27.51(6.42)^{* *}$ & $30.10(6.25)^{* *}$ \\
Turning to religion & $22.7(5.6)$ & $19.86(4.70)^{* *}$ & $22.99(4.67)$ \\
\hline
\end{tabular}

COPE NVI Coping Orientation to the Problems Experienced-New Italian version

$* p \leq 0.05$ compared to mean of general community sample

**Significant difference after Bonferroni correction ( $p \leq 0.01 ; 0.05 / 5)$

${ }^{a}$ Sica et al. [59] problems, and overall accounted for $30.0 \%$ of the variance. In both models, avoidance coping strategies were significant predictors of the outcome (and remained marginally significant after Bonferroni correction), with a positive Beta coeffi Other predictors were not signifi y related to the outcome.

Aggressive behaviour as dependent variable was examined in the second model, which explained $24.6 \%$ of the variance in this scale. Avoidance coping strategies were significant predictors of aggressive behaviour scores (even after Bonferroni correction), with a positive Beta coefficient.

The fourth and final model explained $23.9 \%$ of the variance in total problems. Avoidance coping strategies significantly positively predicted scores at the scale, but not after Bonferroni correction.
Table 5 Significant results of multiple linear regression analyses for ASEBA self-report syndrome scales

\begin{tabular}{llllll}
\hline ASEBA self-report syndrome scales & Predictors & $B$ & $\beta$ & $p$ & $R^{2}$ \\
\hline 1. Anxious/depressed & Age & 0.109 & 0.066 & 0.630 & 0.281 \\
& Intellectual disability & 2.802 & 0.136 & 0.343 & \\
& Self COPE NVI social support & 0.039 & 0.037 & 0.835 & \\
& Self COPE NVI avoidance strategies & 0.518 & 0.358 & $0.016^{*}$ & \\
& Self COPE NVI problem solving & 0.227 & 0.199 & 0.287 & \\
2. Aggressive behaviour & Age & 0.210 & 0.225 & 0.093 & 0.246 \\
& Intellectual disability & 1.677 & 0.145 & 0.294 & \\
3. Internalizing problems & Self COPE NVI avoidance strategies & 0.276 & 0.341 & $0.016^{* *}$ & \\
& Age & 0.276 & 0.114 & 0.395 & 0.300 \\
& Intellectual disability & 1.868 & 0.062 & 0.657 & \\
& Self COPE NVI social support & 0.216 & 0.140 & 0.427 & \\
& Self COPE NVI avoidance strategies & 0.762 & 0.364 & $0.014^{*}$ & \\
& Self COPE NVI problem solving & 0.235 & 0.143 & 0.439 & \\
& Age & 0.270 & 0.125 & 0.372 & 0.239
\end{tabular}

Patients' COPE NVI scores and physical impairments were entered as independent variables on the basis of previous correlation analysis. Age and intellectual disability were included by default as predictors in each regression analysis. $N=51$

ASEBA Achenbach System for Empirically Based Assessment, COPE NVI Coping Orientation to the Problems Experienced-New Italian version

$* p<0.05$

**Significant $p$ values after Bonferroni correction ( $p \leq 0.01$ for model 1,3 and $4 ; p \leq 0.017$ for model 2) 


\section{Regressions performed for parent coping}

Significant results from multiple linear regression analysis examining the contribution of parental coping strategies on patients' emotional behavioural problems $(N=76)$ are presented in Table 6.

The first model explained $24.9 \%$ of the variance in withdrawal-depressive symptoms. Avoidance coping strategies of parents and individual intellectual disability add statistically signifi $y$ to the prediction of the outcome. Both predictors had positive Beta coefficients and were still significant after Bonferroni correction.

The second model considered somatic problems as dependent variable and overall explained $12.6 \%$ of the variance in this scale. Avoidance coping strategies of parents was the only significant predictor of the outcome (positive Beta coefficient) and survived Bonferroni correction.

In the fifth model, considering internalizing problems as dependent variable, the proportion of variance explained was $16.6 \%$. The only signifi predictor of the outcome was individual intellectual disability (positive Beta coeffi which survived Bonferroni correction, whereas avoidance coping strategies of parents showed a trend toward significance. Predictors of thought problems were examined in the third model, which explained $18.9 \%$ of the variance in the dependent variable. Significant predictors of the outcome were individual intellectual disability and parents' social support coping (both with positive Beta coeffi but they did not survive Bonferroni correction.

Rule-breaking behaviour as dependent variable was examined in the fourth model, which explained $19.4 \%$ of the variance in this scale. The variables that significantly contributed to the prediction of scores were cardiomyopathy and problem solving coping strategies of parents, both with negative Beta coefficients that remained significant after Bonferroni correction.

\section{Discussion}

\section{Comments to results}

In the present study, we considered a large population of young patients with MDs, exploring their cognitive and emotional features, the prevalence of neurodevelopmental

Table 6 Significant results of multiple linear regression analyses for ASEBA parent-report syndrome scales

\begin{tabular}{|c|c|c|c|c|c|}
\hline ASEBA parent-report syndrome scales & Predictors & $B$ & $\beta$ & $p$ & $R^{2}$ \\
\hline \multirow[t]{4}{*}{ 1. Withdrawn/depressed } & Age & -0.001 & -0.001 & 0.994 & \multirow[t]{4}{*}{0.249} \\
\hline & Cardiomyopathy & -2.934 & -0.185 & 0.155 & \\
\hline & Intellectual disability & 5.956 & 0.317 & $0.004 * *$ & \\
\hline & Parents' COPE NVI Avoidance strategies & 0.458 & 0.281 & $0.009 * *$ & \\
\hline \multirow[t]{3}{*}{ 2. Somatic complaints } & Age & 0.030 & 0.037 & 0.742 & \multirow[t]{3}{*}{0.126} \\
\hline & Intellectual disability & 1.255 & 0.083 & 0.486 & \\
\hline & Parents' COPE NVI Avoidance strategies & 0.383 & 0.342 & $0.003^{* *}$ & \\
\hline \multirow[t]{4}{*}{ 3. Thought problems } & Age & 0.024 & 0.037 & 0.749 & \multirow[t]{4}{*}{0.189} \\
\hline & Intellectual disability & 2.778 & 0.256 & $0.028 *$ & \\
\hline & Parents’ COPE NVI Social support & 0.172 & 0.269 & $0.021^{*}$ & \\
\hline & Parents' COPE NVI Avoidance strategies & 0.176 & 0.187 & 0.102 & \\
\hline \multirow[t]{4}{*}{ 4. Rule-breaking behaviour } & Age & 0.035 & 0.100 & 0.475 & \multirow[t]{4}{*}{0.194} \\
\hline & Intellectual disability & 0.417 & 0.073 & 0.525 & \\
\hline & Cardiomyopathy & -1.758 & -0.363 & $0.011^{* *}$ & \\
\hline & Parents' COPE NVI Problem solving & -0.122 & -0.314 & $0.007 * *$ & \\
\hline \multirow[t]{4}{*}{ 5. Internalizing problems } & Age & 0.347 & 0.253 & 0.066 & \multirow[t]{4}{*}{0.166} \\
\hline & Intellectual disability & 6.816 & 0.304 & $0.008^{*}$ & \\
\hline & Cardiomyopathy & -3.204 & -0.169 & 0.216 & \\
\hline & Parents' COPE NVI Avoidance strategies & 0.383 & 0.197 & 0.079 & \\
\hline
\end{tabular}

Patient's physical impairments and parents' COPE NVI scores were entered as independent variables on the basis of previous correlation analysis. Age and intellectual disability were included by default as predictors in each regression analysis. $N=76$

$* p<0.05$

**Significant $p$ values after Bonferroni correction ( $p \leq 0.013$ for model 1, 3, 4 and 5; $p \leq 0.017$ for model 2

ASEBA Achenbach System for Empirically Based Assessment, COPE NVI Coping Orientation to the Problems Experienced—New Italian version 
and psychiatric disorders and both their own and their parents coping styles. Furthermore, we assessed the role of individual (age, physical impairment, ID, coping styles) and familial factors (SES, parental coping styles) on psychological outcomes of patients. We used well-validated instruments to collect data, and patients included in the study were all genetically defined.

Our data confirm previous literature and our hypothesis regarding neurodevelopmental disorders such as ID and ASD among patients with DMD: these conditions were found only in the DMD subsample, resulting in $22.3 \%$ of the total sample for ID, and $6.25 \%$ of the total sample for ASD, which are high prevalences if compared to general population $[64,65]$.

In relation to emotional/behavioural problems, as we hypothesized, the first-step screening instruments returned a high rate of internalizing problems, both among young adults (18\%) and-at a major extent-among children (24.6\%) according to ASEBA questionnaires. The overall prevalence of internalizing problems in children resembles that found in our previous study (23.4\%) [15] and in Ricotti and colleagues' study (24\%) [16], which considered a sample of children with DMD similar to our in terms of size and used the same questionnaire. Even exploring SDQ profiles, internalizing problems area (comprising emotional and peer problems scales) was still the highest, and, among children, it was drawn by a serious presence $(17.54 \%)$ of problems with peer relations, as compared to general population of western European countries reported in previous studies [61-63]. These data suggest that children with MDs experience symptoms of anxiety and depression that might be associated with difficulties in gaining peer acceptance and in establishing friendships. In contrast to the aforementioned study by Ricotti and colleagues', we found a very low prevalence of externalizing problems (1.64\% among children and $0 \%$ among young adults, according to ASEBA).

When we observed results of the second-step in-depth diagnostic assessment, we noticed that the percentage of patients meeting clinical caseness is considerably reduced: rates of ADHD, internalizing disorders and conduct/oppositional disorders are in line with prevalence rates reported in general population studies, both for children and for adults (see [66-68] for ADHD; see [60, 69] for internalizing disorders; see [60] for conduct/oppositional disorders). Nevertheless, in children with BMD, we found a much higher rate of ADHD (33\%) compared to children with DMD (1.9\%), LGMD (0\%) or general population data [66]. This observed frequency of ADHD in BMD is similar to that reported by two previous studies $[14,18]$, where a notably higher percentage compared to that observed in DMD has been also replicated [14].
Moreover, comparing the three different types of MDs on overall frequency of emotional/behavioural disorders, BMD subsample was that with the highest rate (23.53\%).

Since prevalence of psychopathology in our overall sample was found to be aligned with that of general population, our interest was directed at the exploration of coping styles of parents and patients, to verify whether they adopt strategies to cope with stressful situations that are protective against psychological distress. Compared to general community, caregivers of patients with MDs relied less on avoidance, problem solving and social support, while they had a higher level of positive attitude. Lower problem solving was also evident in coping profile of patients, together with a lower tendency to turn to religion. These results are in line with our expectations of a grater use of secondary control coping (positive attitude) over primary control coping (problem solving) compared to general population. In previous research on chronic illness, less avoidance attitude and higher acceptance have been related to better psychological adjustment [41, 46]. Based on this knowledge, our observed parental coping profi might be considered adaptive. In a transactional stress and coping model perspective, parents who cope with their children's condition accepting the reality-rather than denying it-and positively reinterpreting the experience, (e.g., through the fi of opportunities for growth), could have a better emotional regulation in the long term, with a positive influence on children's emotional well being.

The lower use of primary control coping (i.e., social support by parents and problem-focusing by both parents and children compared to general population) could lead to different interpretations. Social support could be less used because of family social isolation and stigma. Alternatively, it could be interpreted as a way to adapt; in fact, looking at research evidences on coping with not easily controllable stressors, a high use of coping strategies aimed at directly change the source of stress or the emotional reaction to it (as problem solving and seeking social support), was found to be related to a worse emotional/behavioural adjustment compared to strategies aimed at change aspects of oneself to accommodate to the stressor (such as positive attitude) [41, $61,62]$. These findings do not deny the role of psychological support from the social network in reducing the eff of stress, which can be considered, as described by several studies, a buff between coping with an event and stress [30], but it might be not sufficient on its own to guarantee adjustment [61, 62]. This consideration can be also valid for religious coping, which has not been found to be strongly related to psychological well being [62].

Based on these data, we were further interested to test the association between coping styles and psychological outcomes in our sample, taking also into account individual variables. The results of the regression analyses consistently 
confirm our hypotheses, showing the use of avoidant coping strategies to be associated with higher risk of emotional/ behavioural problems in patients with MDs. A higher risk of psychological distress was observed in patients with MDs both when avoidant coping was used by their parents and when they were themselves to use it. These findings support the aforementioned past study of Thompson and colleagues [34], which indicated higher parent use of disengagement coping to be associated with poor adjustment in children with DMD, as well as previous studies on chronically ill patients where their self-reported use of avoidance was consistently found to be associated with psychological distress, especially anxiety and depression [40]. In our sample, parents who were more prone to use avoidant coping were more likely to have children with increased levels of internalizing problems. On the other hand, if were patients themselves to make a greater use of avoidant coping strategies, they were more likely to report higher levels of both internalizing (i.e., anxiety, depression) and externalizing problems (i.e., aggressive behaviour).

We also noticed that higher use of social support from parents was associated with an increase in children's thought problems, supporting the hypothesis that this coping strategy may not always be useful, as it could reflect a tendency to vent emotions, which is indeed a form of disengagement [58]. However, caution is needed in interpreting this result, because after Bonferroni correction, the p value was far from signifi level. On the other hand, we found less rule-breaking problems in patients whose parents used more problem-focused coping, suggesting a possible protective role of this strategy, especially when it is used for the sources of stress perceived as more controllable, such as problems related to school or home help or providing adaptive equipment. No such association was found for problem solving when used by patients, in line with mixed findings regarding this strategy reported in literature $[40,58]$.

Observing parents' rating of their childrens' psychological difficulties, regression analyses also showed that patients' intellectual level was related to their emotional/behavioural outcome: the presence of comorbid ID was in fact related with increased internalizing problems and thought problems. This is consistent with numerous studies indicating ID as a risk factor for developing psychopathology [70, 71].

Finally, it is interesting to note that measures of disease progression (age and wheelchair use) did not contribute significantly to reported elevated behaviour problems, confirming previous evidences suggesting coping style and adjustment as more salient to psychological outcome than disease severity [72, 73].

\section{Strengths and weaknesses of the study}

This study adds to current knowledge regarding neurodevelopmental disorders and psychological status among people with MDs. It also provides new knowledge on how families cope with stress and on the relationship that specific coping strategies and clinical aspects of MDs have with the risk of psychological distress. We consider strengths of our study the use of a genetically well-defined clinical population and of validated instruments directly administered to both patients and their parents.

However, results should be interpreted with some limitations in mind.

First, even though this sample is appropriate for a monocentric study, our three subgroups of MDs were not homogeneous in terms of size (the majority had DMD), whereas to perform more precise group comparisons, larger and more balanced sample sizes will be needed. This limitation also concern comparisons of coping strategies between parents and general population or patients and general population.

Moreover, since the COPE questionnaire is not validated for children, we needed to exclude them from the investigation of patients' self-reported coping styles. In future studies, it will be desirable to address this limitation.

In the second-step, in-depth diagnostic assessment, additional structured interviews (i.e., ADOS and DAWBA) were administered only in a subsample of 38 patients (34.2\% of the total sample), according to our assessment procedure: this may have led to an underestimation of the true rate of psychopathology in this special clinical population. Nevertheless, the utility of behavioural screening instruments for risk stratifi has been largely demonstrated [74, 75]. Sheldrick and colleagues [75] reported that children who screened positive at CBCL 6-18 and SDQ were approximately 2-5 times more likely to qualify for a psychiatric diagnosis compared to those children who screened negative.

Since the study has a cross-sectional design, causality cannot be fully investigated and interpretation of results needs caution.

Finally, the large number of statistical tests performed on the same data set may have increased the probability of statistically significant results by chance. To control this risk, we have applied Bonferroni correction to multiple analyses.

\section{Future research}

Future research should use larger and more balanced sample sizes to draw more defi e conclusions and to perform more precise group comparisons. In particular, it would be interesting to extend BMD sample to better explore its neurodevelopmental and emotional profile, since there are suggestions of a greater vulnerability to psychopathology 
among BMD patients. Moreover, designing and conducting longitudinal studies can improve understanding the role of parental coping later in emotional/behavioural development of children. Finally, it would be interesting to explore coping strategies in younger children, with less than 16 years. To do this, it will be desirable to administer instruments for the study of coping specifically designed for children and with Italian normative data.

\section{Conclusions}

In conclusion, our study indicates that, despite the relatively high presence of neurodevelopmental disorders (i.e., ASD, ID, ADHD) and internalizing symptoms, children and young adults with MDs exhibit rates of diagnosed psychopathology that are in line with those of general population. Within families of our sample, parents face stress through the use of a coping style known to be more adaptive in the context of a chronic illness, wherein positive reinterpretation and growth and acceptance prevail over denial and behavioural or mental disengagement. Findings of this research also show a link between coping strategies and psychological adjustment in patients with MDs. The most consistent relationship was with avoidance, that, when used by parents, predicts internalizing symptoms in patients, and, when used by patients themselves, predicts both internalizing and externalizing symptoms. Moreover, the comorbid presence of ID is indicated as an additional risk factor associated with worse emotional and behavioural outcomes, while disease progression is not. Given the high prevalence of internalizing symptomatology reported in children and young adults with MDs, results of this study suggest that interventions targeting both parents and patients, aimed at discouraging use of avoidant coping and, on the other hand, promoting use of adaptive coping (i.e., positive reinterpretation, acceptance, problem solving for situations that are controllable) could reducevia indirect and direct mechanisms - anxiety and depression and, more generally, psychological distress among patients.

Acknowledgements The authors thank all patients and families for their collaboration and the medical, nursing and technical personnel of the IRCCS E Medea involved in the care and evaluation of the patients (E. Mornati, C. Gerosa, S. Molteni).

Funding The work was supported by Grants of the Italian Ministry of Health Ricerca Corrente 2018 and 5 per mille funds for biomedical research (Grant no. 2013-2016).

\section{Compliance with ethical standards}

Conflicts of interest The authors declare that they have no conflict of interest.
Ethical standards The study was approved by the Ethical Committee of the Scientific Institute IRCCS Eugenio Medea according to the Declaration of Helsinki.

Informed consent All patients provided written informed consent for participation in the study.

\section{References}

1. Carter GT (1997) Rehabilitation management in neuromuscular disease. J Neuro Rehabil 11:69-80. https://doi.org/10.1177/15459 6839701100201

2. Pieterse AJ, Cup EH, Knuijt S et al (2008) Development of a tool to guide referral of patients with neuromuscular disorders to allied health services. Part two. Disabil Rehabil 30:863-870. https://doi. org/10.1080/09638280701403460

3. Straub V, Bertoli M (2016) Where do we stand in trial readiness for autosomal recessive limb girdle muscular dystrophies? Neuromuscul Disord 26:111-125. https://doi.org/10.1016/j. nmd.2015.11.012

4. Birnkrant DJ, Bushby K, Bann CM et al (2018) Diagnosis and management of Duchenne muscular dystrophy, part 2: respiratory, cardiac, bone health, and orthopaedic management. Lancet Neurol 17:347-361. https://doi.org/10.1016/S1474-4422(18)30025-5

5. Birnkrant DJ, Bushby K, Bann CM et al (2018) Diagnosis and management of Duchenne muscular dystrophy, part 3: primary care, emergency management, psychosocial care, and transitions of care across the lifespan. Lancet Neurol 17:445-455. https://doi. org/10.1016/S1474-4422(18)30026-7

6. Birnkrant DJ, Bushby K, Bann CM et al (2018) Diagnosis and management of Duchenne muscular dystrophy, part 1: diagnosis, and neuromuscular, rehabilitation, endocrine, and gastrointestinal and nutritional management. Lancet Neurol 17:251-267. https:// doi.org/10.1016/S1474-4422(18)30024-3

7. Fayssoil A, Abasse S, Silverston K (2017) Cardiac involvement classification and therapeutic management in patients with duchenne muscular dystrophy. J Neuromuscul Dis 4:17-23. https://doi. org/10.3233/JND-160194

8. Magri F, Nigro V, Angelini C et al (2017) The Italian limb girdle muscular dystrophy registry: relative frequency, clinical features, and diff ential diagnosis. Muscle Nerve 55:55-68. https://doi. org/10.1002/mus.25192

9. Taghizadeh E, Rezaee M, Barreto GE, Sahebkar A (2019) Prevalence, pathological mechanisms, and genetic basis of limb-girdle muscular dystrophies: a review. J Cell Physiol 234:7874-7884. https://doi.org/10.1002/jcp.27907

10. Mercuri E, Muntoni F (2013) Muscular dystrophies. Lancet 381:845-860. https://doi.org/10.1016/S0140-6736(12)61897-2

11. Pousada T, Groba B, Nieto-Riveiro L et al (2018) Determining the burden of the family caregivers of people with neuromuscular diseases who use a wheelchair. Medicine (Baltimore) 97:e11039. https://doi.org/10.1097/MD.0000000000011039

12. Snow WM, Anderson JE, Jakobson LS (2013) Neuropsychological and neurobehavioral functioning in Duchenne muscular dystrophy: a review. Neurosci Biobehav Rev 37:743-752. https://doi. org/10.1016/j.neubiorev.2013.03.016

13. Darke J, Bushby K, Le Couteur A, McConachie H (2006) Survey of behaviour problems in children with neuromuscular diseases. Eur J Paediatr Neurol 10:129-134. https://doi.org/10.1016/j. ejpn.2006.04.004

14. Latimer R, Street N, Conway KC (2017) Secondary conditions among males with duchenne or becker muscular dystrophy. J 
Child Neurol 32:663-670. https://doi.org/10.1177/0883073817 701368

15. Colombo P, Nobile M, Tesei A et al (2017) Assessing mental health in boys with Duchenne muscular dystrophy: emotional, behavioural and neurodevelopmental profi in an Italian clinical sample. Eur J Paediatr Neurol 21:639-647. https://doi. org/10.1016/j.ejpn.2017.02.007

16. Ricotti V, Mandy WP, Scoto M et al (2016) Neurodevelopmental, emotional, and behavioural problems in Duchenne muscular dystrophy in relation to underlying dystrophin gene mutations. Dev Med Child Neurol 58:77-84. https://doi.org/10.1111/dmcn.12922

17. Banihani R, Smile S, Yoon G et al (2015) Cognitive and neurobehavioral profile in boys with duchenne muscular dystrophy. J Child Neurol 30:1472-1482. https://doi.org/10.1177/0883073815 570154

18. Young HK, Barton BA, Waisbren S et al (2008) Cognitive and psychological profile of males with Becker muscular dystrophy. J Child Neurol 23:155-162. https://doi.org/10.1177/0883073807 307975

19. Pane M, Lombardo ME, Alfi i P et al (2012) Attention deficit hyperactivity disorder and cognitive function in Duchenne muscular dystrophy: phenotype-genotype correlation. J Pediatr 161:705-9.e1. https://doi.org/10.1016/j.jpeds.2012.03.020

20. Caspers Conway K, Mathews KD, Paramsothy P et al (2015) Neurobehavioral concerns among males with dystrophinopathy using population-based surveillance data from the muscular dystrophy surveillance, tracking, and research network. J Dev Behav Pediatr 36:455-463. https://doi.org/10.1097/DBP.0000000000000177

21. Miladi N, Bourguignon JP, Hentati F (1999) Cognitive and psychological profi e of a Tunisian population of limb girdle muscular dystrophy. Neuromuscul Disord 9:352-354. https://doi. org/10.1016/S0960-8966(99)00006-1

22. Magliano L, D’Angelo MG, Vita G et al (2014) Psychological and practical diffi among parents and healthy siblings of children with Duchenne vs. Becker muscular dystrophy: an Italian comparative study. Acta Myol 33:136-143

23. Aho AC, Hultsjö S, Hjelm K (2015) Young adults experiences of living with recessive limb-girdle muscular dystrophy from a salutogenic orientation: an interview study. Disabil Rehabil 37:2083-2091. https://doi.org/10.3109/09638288.2014.998782

24. Sabharwal R (2014) The link between stress disorders and autonomic dysfunction in muscular dystrophy. Front Physiol. https:// doi.org/10.3389/fphys.2014.00025

25. Gagliardi BA (1991) The impact of Duchenne muscular dystrophy on families. Orthop Nurs 10:41-49. https://doi.org/10.1097/00006 416-199109000-00009

26. Kenneson A, Bobo JK (2010) The effect of caregiving on women in families with Duchenne/Becker muscular dystrophy. Health Soc Care Commun 18:520-528. https://doi.org/10.111 1/j.1365-2524.2010.00930.x

27. Pangalila RF, van den Bos GA, Stam HJ et al (2012) Subjective caregiver burden of parents of adults with Duchenne muscular dystrophy. Disabil Rehabil 34:988-996. https://doi. org/10.3109/09638288.2011.628738

28. Holroyd J, Guthrie D (1986) Family stress with chronic childhood illness: cystic fibrosis, neuromuscular disease, and renal disease. J Clin Psychol 42:552-561. https://doi. org/10.1002/1097-4679(198607)42:4\%3c552:AID-JCLP227042 0403\%3e3.0.CO;2-8

29. Abi Daoud MS, Dooley JM, Gordon KE (2004) Depression in parents of children with Duchenne muscular dystrophy. Pediatr Neurol 31:16-19. https://doi.org/10.1016/j.pediatrneurol.2004.01.011

30. Magliano L, Patalano M, Sagliocchi A et al (2014) "I have got something positive out of this situation": psychological benefits of caregiving in relatives of young people with muscular dystrophy. $\mathrm{J}$ Neurol 261:188-195. https://doi.org/10.1007/s00415-013-7176-8
31. Pangalila RF, Van den Bos GA, Bartels B et al (2015) Quality of life of adult men with Duchenne muscular dystrophy in the Netherlands: implications for care. J Rehabil Med 47:161-166. https://doi.org/10.2340/16501977-1898

32. Landfeldt E, Lindgren P, Bell CF et al (2016) Health-related quality of life in patients with Duchenne muscular dystrophy: a multinational, cross-sectional study. Dev Med Child Neurol 58:508-515. https://doi.org/10.1111/dmcn.12938

33. Bendixen RM, Senesac C, Lott DJ, Vandenborne K (2012) Participation and quality of life in children with Duchenne muscular dystrophy using the International Classification of Functioning, Disability, and Health. Health Qual Life Outcomes. https://doi. org/10.1186/1477-7525-10-43

34. Thompson RJ (1985) Coping with the stress of chronic childhood illness. In: O’Quinn A (ed) Management of chronic disorders of childhood. G. K. Hall, Boston, pp 11-41

35. Thompson RJ, Gustafson KE (1996) Adaptation to chronic childhood illness. American Psychological Association, Washington DC

36. Lazarus RS, Folkman S (1984) Stress appraisal and coping. Springer, New York

37. Carver CS, Connor-Smith J (2010) Personality and coping. Annu Rev Psychol 61:679-704. https://doi.org/10.1146/annurev.psych .093008 .100352

38. Eisenbarth C (2012) Coping profiles and psychological distress: a cluster analysis. N Am J Psychol 14:485-496

39. Nielsen MB, Knardahl S (2014) Coping strategies: a prospective study of patterns, stability, and relationships with psychological distress. Scand J Psychol 55:142-150. https://doi.org/10.1111/ sjop.12103

40. Compas BE, Jaser SS, Dunn MJ, Rodriguez EM (2012) Coping with chronic illness in childhood and adolescence. Annu Rev Clin Psychol 8:455-480. https://doi.org/10.1146/annurev-clinp sy-032511-143108

41. Spira AP, Zvolensky MJ, Eifert GH, Feldner MT (2004) Avoidance-oriented coping as a predictor of panic-related distress: a test using biological challenge. J Anxiety Disord 18:309-323. https:// doi.org/10.1016/S0887-6185(02)00249-9

42. Anshel MH, Kim KW, Kim BH, Chang KJ, Eom HJ (2001) A model for coping with stressful events in sport: theory, application, and future directions. Int J Sport Psychol 32:43-75

43. Thompson RJ Jr, Zeman JL, Fanurik D, Sirotkin-Roses M (1992) The role of parent stress and coping and family functioning in parent and child adjustment to Duchenne Muscular Dystrophy. J Clin Psychol 48:11-19. https://doi.org/10.1002/1097-4679(19920 1)48:1\%3c11:AID-JCLP2270480103\%3e3.0.CO;2-4

44. Steinberg L, Cauffman E, Woolard J, Graham S, Banich M (2009) Are adolescents less mature than adults?: minors' access to abortion, the juvenile death penalty, and alleged APA “flip-flop”. Am Psychol 64:583-594. https://doi.org/10.1037/a0014763

45. Bushby K, Finkel R, Birnkrant DJ et al (2010) Diagnosis and management of Duchenne muscular dystrophy, Part 2: implementation of multidisciplinary care. Lancet Neurol 9:177-189. https://doi. org/10.1016/S1474-4422(09)70272-8

46. Vissing J (2016) Limb girdle muscular dystrophies: classifi tion, clinical spectrum and emerging therapies. Curr Opin Neurol 29:635-641. https://doi.org/10.1097/WCO.0000000000000375

47. Padberg GW, van Engelen BG (2009) Facioscapulohumeral muscular dystrophy. Curr Opin Neurol 22:539-542. https://doi. org/10.1097/WCO.0b013e328330a572

48. Hollingshead AB (1975) Four factor index of socioeconomic status. Yale J Sociol 8:21-52

49. Achenbach TM, Rescorla L (2001) Manual for the ASEBA schoolage forms \& profi child behavior checklist for ages 6-18, teacher's report form, youth self-report. An Integrated System 
of Multi-Informant Assessment. Research Center for Children, Youth, \& Families, University of Vermont, Burlington

50. Achenbach TM, Rescorla L (2003) Manual for the ASEBA adult forms and profiles. Research Center for Children, Youth, \& Families, University of Vermont, Burlington

51. Goodman R (1999) The extended version of the Strengths and Difficulties Questionnaire as a guide to child psychiatric caseness and consequent burden. J Child Psychol Psychiatry 40:791-799. https://doi.org/10.1017/S0021963099004096

52. Stone LL, Otten R, Engels RC et al (2010) Psychometric properties of the parent and teacher versions of the strengths and difficulties questionnaire for 4- to 12-year-olds: a review. Clin Child Fam Psychol Rev 13:254-274. https://doi.org/10.1007/s1056 7-010-0071-2

53. Goodman R (1997) The strengths and difficulties questionnaire: a research note. J Child Psychol Psychiatry 38:581-586. https:// doi.org/10.1111/j.1469-7610.1997.tb01545.x

54. Goodman R, Ford T, Richards H et al (2000) The development and well-being assessment: description and initial validation of an integrated assessement of child and adolescent psychopathology. J Child Psychol Psychiatry 41:645-655. https://doi.org/10.1017/ S0021963099005909

55. Lord C, Rutter M, DiLavore PC, Risi S (1999) ADOS. Autism diagnostic observation schedule. Manual. WPS, Los Angeles

56. Ford T, Goodman R, Meltzer H (2003) The British child and adolescent mental health survey 1999: the prevalence of DSM-IV disorders. J Am Acad Child Adolesc Psychiatry 42:1203-1211. https://doi.org/10.1097/00004583-200310000-00011

57. Goodman A, Heiervang E, Collishaw S, Goodman R (2011) The "DAWBA bands" as an ordered-categorical measure of child mental health: description and validation in British and Norwegian samples. Soc Psychiatry Psychiatr Epidemiol 6:521-532. https:// doi.org/10.1007/s00127-010-0219-x

58. Carver CS, Scheier MF, Weintraub KJ (1989) Assessing coping strategies: a theoretically based approach. J Pers Soc Psychol 56:267-283. https://doi.org/10.1037/0022-3514.56.2.267

59. Sica C, Magni C, Ghisi M et al (2008) Coping orientation to problems experienced-Nuova Versione Italiana (COPE-NVI): Uno strumento per la misura degli stili di coping. Psicoterapia Cognitiva E Comportamentale 14:27-53

60. Frigerio A, Rucci P, Goodman R et al (2009) Prevalence and correlates of mental disorders among adolescents in Italy: the PrISMA study. Eur Child Adolesc Psychiatry 18:217-226. https ://doi.org/10.1007/s00787-008-0720-x

61. Meltzer H, Gatward R, Goodman R, Ford T (2003) Mental health of children and adolescents in Great Britain. Int Rev Psychiatry 15:185-187. https://doi.org/10.1080/0954026021000046155

62. Rothenberger A, Becker A, Erhart M et al (2008) Psychometric properties of the parent strengths and difficulties questionnaire in the general population of German children and adolescents: results of the BELLA study. Eur Child Adolesc Psychiatry 17:99105. https://doi.org/10.1007/s00787-008-1011-2
63. Kovess-Masfety V, Husky MM, Keyes K et al (2016) Comparing the prevalence of mental health problems in children 6-11 across Europe. Soc Psychiatry Psychiatr Epidemiol 51:1093-1103. https ://doi.org/10.1007/s00127-016-1253-0

64. Narzisi A, Posada M, Barbieri F et al (2018) Prevalence of Autism Spectrum Disorder in a large Italian catchment area: a schoolbased population study within the ASDEU project. Epidemiol Psychiatr Sci 6:1-10. https://doi.org/10.1017/S20457960180004 83

65. McKenzie K, Milton M, Smith G, Ouellette-Kuntz H (2016) Systematic review of the prevalence and incidence of intellectual disabilities: current trends and issues. Curr Dev Disord Rep 3:104-115. https://doi.org/10.1007/s40474-016-0085-7

66. Polanczyk G, De Lima MS, Horta BL et al (2007) The worldwide prevalence of ADHD: a systematic review and metaregression analysis. Am J Psychiatry 164:942-948. https://doi.org/10.1176/ ajp.2007.164.6.942

67. Fayyad J, De Graaf R, Kessler R et al (2007) Cross-national prevalence and correlates of adult attention-deficit hyperactivity disorder. Br J Psychiatry 190:402-409. https://doi.org/10.1192/ bjp.bp.106.034389

68. Simon V, Czobor P, Bálint S et al (2009) Prevalence and correlates of adult attention-deficit hyperactivity disorder: metaanalysis. Br J Psychiatr 194:204-211. https://doi.org/10.1192/ bjp.bp.107.048827

69. Steel Z, Marnane C, Iranpour C et al (2014) The global prevalence of common mental disorders: a systematic review and meta-analysis 1980-2013. Int J Epidemiol 43:476-493. https:// doi.org/10.1093/ije/dyu038

70. Wallander JL, Dekker MC, Koot HM (2003) Psychopathology in children and adolescents with intellectual disability: measurement, prevalence, course, and risk. Int Rev Res Ment Retard 26:93-134. https://doi.org/10.1016/S0074-7750(03)01003-6

71. Condly SJ (2006) Resilience in children: a review of literature with implications for education. Urban Educ 41:211-236. https ://doi.org/10.1177/0042085906287902

72. Nereo NE, Fee RJ, Hinton VJ (2003) Parental stress in mothers of boys with Duchenne muscular dystrophy. J Pediatr Psychol 28:473-484. https://doi.org/10.1093/jpepsy/jsg038

73. Fee RJ, Hinton VJ (2011) Resilience in children diagnosed with a chronic neuromuscular disorder. J Dev Behav Pediatr 32:644-650. https://doi.org/10.1097/DBP.0b013e318235d614

74. Carter AS, Briggs-Gowan MJ, Davis NO (2004) Assessment of young children's social-emotional development and psychopathology: recent advances and recommendations for practice. J Child Psychol Psychiatry 45:109-134. https://doi.org/10.104 6/j.0021-9630.2003.00316.x

75. Sheldrick RC, Benneyan JC, Kiss IG et al (2015) Thresholds and accuracy in screening tools for early detection of psychopathology. J Child Psychol Psychiatry 56:936-948. https://doi. org/10.1111/jcpp.12442 